\title{
Brownian dynamics simulation study on force-velocity relation in actin-based membrane protrusion
}

\author{
Yasuhiro Inoue $^{1,2}$ - Takeji Deji ${ }^{3}$ - Taiji Adachi ${ }^{1}$
}

Received: 15 January 2015 / Revised: 26 May 2015 / Accepted: 30 May 2015 / Published online: 6 June 2015

(C) OWZ 2015

\begin{abstract}
In actin polymerization-based membrane protrusions, the velocity of the protruding membrane decreases with increasing opposing force. This relationship, known as the force-velocity relation (FVR), can be concave up or concave down. However, the mechanism by which FVR exhibits two trends remains debatable. In this study, we simulate the Brownian dynamics of the protruding membrane driven by actin polymerization. The actin diffusion coefficient is retained constant, and the actin polymerization rates are set to low (case-L) or high (case-H). The protruding membrane in the case- $\mathrm{L}$ and case- $\mathrm{H}$ simulations exhibits a concave up and concave down FVR, respectively. The polymerization rate affects the spatial distribution of actins, and thereby their physical contact with the membrane. The membrane is predominantly sustained by monomers in case- $\mathrm{L}$ and filaments in case-H. This suggests that membrane protrusions respond differently to the opposing force because actins are differently distributed around the membrane.
\end{abstract}

Keywords Actin-based motility - Brownian dynamics simulation $\cdot$ Force-velocity relation

Yasuhiro Inoue

inoue@frontier.kyoto-u.ac.jp

Taiji Adachi

adachi@frontier.kyoto-u.ac.jp

1 Institute for Frontier Medical Sciences, Kyoto University, Kyoto, Japan

2 CREST, JST, Saitama, Japan

3 Department of Mechanical Engineering and Science, Kyoto University, Kyoto, Japan

\section{Introduction}

Lamellipodium protrusions of migrating cells [1-3], propulsive movements of Listeria monocytogenes [4], and small beads coated with actin-polymerization activators [5] exhibit actin-based motility, which generates force by the growth of actin filaments against an obstacle [6-8]. The force generated by actin polymerizations is characterized using the forcevelocity relation (FVR) which is defined as the decrease in the velocity of the protruding obstacle with increasing opposing force. FVR has been directly measured in experiments, and reported as two types-concave up [5] and concave down [9]. To explain the mechanism of actin-based motility, researchers have developed several theoretical models, including the well-known Brownian ratchet (BR) [10-12]. The BR assumes that polymerized actin filaments beneath the obstacle rectify the Brownian motion of the obstacle, generating a unidirectional force; the resulting FVR is concave up. BR prediction confirms the measurements of the concave-up FVR for the protrusive movements of small beads coated with actin-polymerization activators [5], movements of L. monocytogenes [12], and the filopodium protrusions of migrating cells [13], but BR model cannot predict the concave-down FVR of the lamellipodia of migrating cells [9]. Concave-down FVRs have been reproduced by the computation models of cell motility [14] and the Brownian dynamics (BD) simulations of the actin-based motility of a disk [15], both of which consider actin filament branches. One specific cytoskeletal structure in lamellipodia is the actin branched network [16]. The Arp2/3 complex binds to the side of an existing filament, initiates actin branch nucleation [17] and increases the number of filaments in the vicinity of the protruding membrane or obstacle. Thus, the membrane protrusion is effectively supported against higher load by densely branched networks in lamellipodia. In addition to 
the chemical pathway of the branch formation by Arp $2 / 3$ [18], Arp2/3-branch nucleation is mechanosensitive as the favored response to filament bending [19], which may also lead to a concave-down FVR.

In this study, we investigate the reason behind the emergence of two types of FVR. Herein, we focus on the effects of multiple filaments and the time-scale ratio of polymerization to monomer diffusion. We do not consider filament branching, which increases the number of filaments in the time course. Next, we examine the manner in which the shape of the FVR depends on the polymerization rate under loads sustained by multiple filaments. We explicitly express the motions of filaments and monomers in BD simulations and examine the protrusive movement of a flat membrane driven by actin polymerization. Our numerical simulations reproduce concave-up and -down FVRs, depending on the spatial distribution of actins in the membrane vicinity.

\section{Mathematical model}

\subsection{Motion of actin}

To simulate a protruding membrane driven by actin polymerization, it is desirable to have mathematical models that capture not only membrane motion but also the microscopic motion and kinetics of actin. Thus, in this study, we express the actin dynamics by a BD model [20,21], which treats actin monomers as spherical particles. The $\mathrm{BD}$ equation describes the motion of actin particle $i$ as follows.

$\gamma \mathbf{v}_{i}=-\nabla_{i} U_{i}-\nabla_{i} U_{i}^{\mathrm{m}}+\xi$.

Here, $\gamma$ is the friction constant and $\xi$ is a random force vector, $\mathbf{v}_{i}$ is the velocity vector of actin particle $i$, and $\nabla_{i}$ denotes a vector differentiation with respect to the position vector of actin particle $i$. The random force satisfies two conditions:

$\left\langle\xi(t) \xi\left(t^{\prime}\right)\right\rangle=2 \gamma k_{\mathrm{B}} T \delta_{\alpha \beta} \delta\left(t-t^{\prime}\right)$,

$\langle\xi(t)\rangle=\mathbf{0}$,

where $k_{B}$ and $T$ are the Boltzmann constant and temperature of the system, respectively. $\delta_{\alpha \beta}$ denotes the Kronecker delta. Subscripts $\alpha$ and $\beta$ indicate the $\alpha$ - and $\beta$-components of the vector, respectively. $\delta\left(t-t^{\prime}\right)$ is the Dirac delta function. The potential energy $U_{i}$ of the actin particle $i$ is the sum of interaction energies, which is described as

$U_{i}=\sum_{i^{\prime}} U_{i i^{\prime}}^{\text {length }}+U_{i}^{\text {angle }}+\sum_{k} U_{i k}^{\text {excl }}$.
The interaction between the actin particles in an actin filament is described in terms of their potential energies as

$U_{i i^{\prime}}^{\text {length }}=\frac{K_{\mathrm{t}}}{2}\left(l_{i i^{\prime}}-l_{i i^{\prime}}^{\mathrm{eq}}\right)^{2}$,

and

$U_{i}^{\mathrm{angle}}=K_{\mathrm{be}}\left[1-\cos \left(\theta_{i}-\theta_{i}^{\mathrm{eq}}\right)\right]$,

where actin particle $i^{\prime}$ is a neighbor of actin particle $i$ in the filament, i.e., $i^{\prime}=i+1$ or $i-1$. $U_{i i^{\prime}}^{\text {length }}$ is the elastic energy stored in a spring of length $l_{i i^{\prime}}$ between actin particles $i$ and $i^{\prime}$. Superscript "eq" indicates the stress free state. $K_{\mathrm{t}}$ indicates the spring constant. The bending potential energy $U_{i}^{\text {angle }}$ depends on the angle $\theta_{i}$ formed between three actin particles $i-1, i$, and $i+1$ across two springs in the filament. $K_{\text {be }}$ denotes the bending rigidity.

The volume of the actin particle is expressed as follows in terms of the truncated Lennard-Jones potential $U_{i k}^{\text {excl }}$ between two arbitrary actin particles $i$ and $k$ :

$U_{i k}^{\mathrm{excl}}\left(d_{i k}\right)=\left\{\begin{array}{ll}U_{L J}\left(d_{i k}\right) & \text { for } d_{i k} \leq d_{c} \\ U_{L J}\left(d_{c}\right) & \text { for } d_{i k}>d_{c}\end{array}\right.$,

where

$$
\begin{aligned}
U_{L J}\left(d_{i k}\right) & =4 \epsilon\left[\left(\frac{\sigma}{d_{i k}}\right)^{12}-\left(\frac{\sigma}{d_{i k}}\right)^{6}\right], \\
d_{c} & =\sqrt[6]{2} \sigma
\end{aligned}
$$

Here $d_{i k}$ is the distance between actin particles $i$ and $k . \epsilon$ and $\sigma$ are the energy coefficient and the exclusion size of the actin particle, respectively.

The potential energy $U_{i}^{\mathrm{m}}$ expresses the repulsive interaction between actin particle $i$ and the membrane using the Lennard-Jones potential:

$U_{i}^{\mathrm{m}}=\left\{\begin{array}{ll}U_{L J}\left(\left|z_{i}-z_{\mathrm{m}}\right|\right) & \text { for }\left|z_{i}-z_{\mathrm{m}}\right| \leq d_{c} \\ U_{L J}\left(d_{c}\right) & \text { for }\left|z_{i}-z_{\mathrm{m}}\right|>d_{c}\end{array}\right.$.

Here, $z_{i}$ and $z_{\mathrm{m}}$ are the $z$-components of the position vector of actin particle $i$ and the membrane, respectively.

Actin monomers are explicitly modeled as the actin particles not connected to any filaments. Thus, the motion of a free actin particle $j$ is given by

$\gamma \mathbf{v}_{j}=-\sum_{k} \nabla_{j} U_{j k}^{\mathrm{excl}}-\nabla_{j} U_{j}^{\mathrm{m}}+\xi$ 


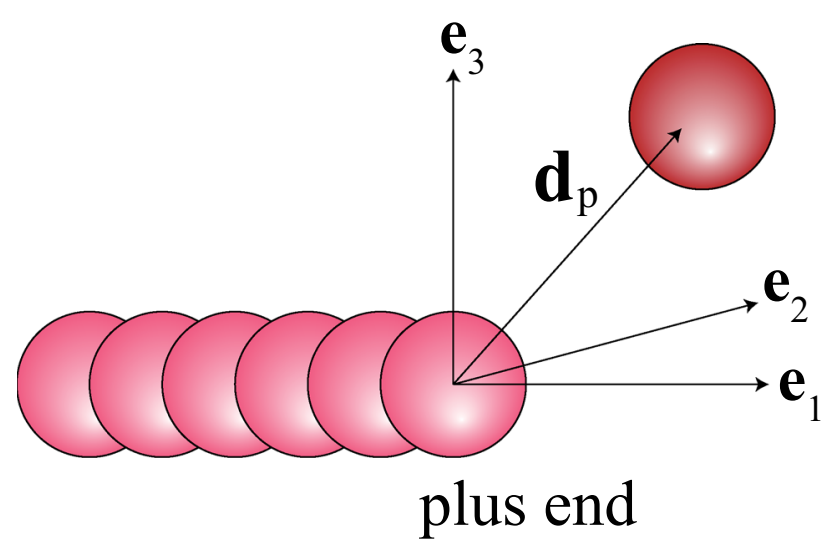

Fig. 1 Schematic of the kinetics model of actin polymerization. An orthogonal coordinate system $\left(O-x_{1}, x_{2}, x_{3}\right)\left(x_{1} \geq 0\right)$ whose origin is set at the center of the actin particle at the plus end, and its base vectors $\left(\mathbf{e}_{1}, \mathbf{e}_{2}, \mathbf{e}_{3}\right)$, where the actin filament is elongated along the $x_{1}$ direction

\subsection{Kinetics of actin polymerization}

Polymerization by bonding an actin molecule to the plus end of the filament is a fundamental process of filament elongation. Here, we adopt a stochastic polymerization model that accounts for filament interference (responsible for the asymmetric accessibility of particles to the plus end) and the diffusion-limited nature of the polymerization kinetics [21]. We construct an orthogonal coordinate system $\left(O-x_{1}, x_{2}, x_{3}\right)\left(x_{1} \geq 0\right)$ with the origin at the center of the actin particle at the plus end, and denote its base vectors by $\left(\mathbf{e}_{1}, \mathbf{e}_{2}, \mathbf{e}_{3}\right)$, where the $x_{1}$ axis is the elongation direction (see Fig. 1). To express the asymmetric accessibility of particles to the plus end and the diffusion-limited nature of the polymerization kinetics, we introduce a polymerization angle criterion $\phi_{c}$ and a vector $\mathbf{d}_{\mathrm{p}}$ that defines the relative position of a candidate actin particle for polymerization to the plus end particle of the actin filament. Polymerization is triggered with conditional probability $p$ if the following conditions hold:

$\left|\mathbf{d}_{p}\right| \leq \sigma$,

$\arccos \left(\frac{\mathbf{d}_{\mathrm{p}} \cdot \mathbf{e}_{1}}{\left|\mathbf{d}_{\mathrm{p}}\right|}\right) \leq \phi_{c}$.

Then, by assuming the spring connection, we model polymerization by binding the actin particle to the plus end particle.

Given the diffusion limited nature of the actin polymerization, we characterize the actin dynamics by a dimensionless number, $\alpha$, defined as the ratio of the polymerization time to the diffusion time:

$\alpha=\frac{\tau_{p}}{\tau_{d}}$

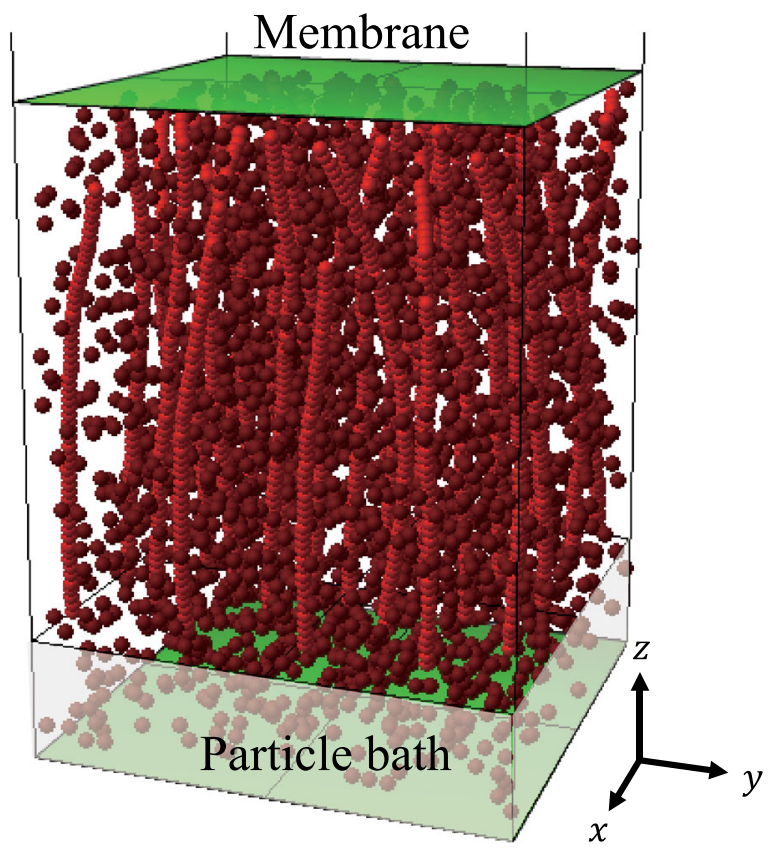

Fig. 2 Schematic of the simulation set-up and definition of the coordinate system

Here, the diffusion time $\tau_{d}$ and polymerization time $\tau_{p}$ are respectively defined by

$$
\begin{aligned}
\tau_{d} & =\frac{h^{2}}{2 D}, \\
\tau_{p} & =\frac{1}{K_{\mathrm{on} \rho}} .
\end{aligned}
$$

The diffusion constant of actin $D$ is given by the Einstein relation $D=k_{B} T / \gamma$. The polymerization rate $K_{\text {on }}$ was numerically obtained in BD simulations of a single actin filament with free actin monomers of density $\rho$ binding with conditional probability $p$.

The polymerization of one monomer incrementally extends the actin filament by $h=\sigma / 2$ [11]. The ratio $\alpha$ is the mean time interval between polymerization events, which is relative to the time scale of monomer diffusion.

\subsection{Membrane motion}

Because membrane flexibility does not change the qualitative trend of FVR (concave up) [13], we ignore the membrane deformation induced by actin polymerization and focus on the conditions that alter the type of FVR. For simplicity, we regard the membrane as a flat plate with area $A$, and consider its motion along the $z$-axis only (Fig. 2). The equation of motion of the membrane is given by 
$\gamma_{\mathrm{mem}} v_{\mathrm{mem}}=-\sum_{k} \nabla U_{k}^{\mathrm{m}}-F-F_{b}+\xi_{\mathrm{mem}}$.

The left hand side of Eq. (17) is the frictional force, computed as the product of the friction coefficient $\gamma_{\text {mem }}$ and the velocity $v_{\text {mem }}$ along the $\mathrm{z}$-axis. On the right hand side, the potential energy $U_{k}^{\mathrm{m}}$ is the repulsive interaction between the membrane and actin $k$, which is computed by Eq. (10). Here, $\nabla$ denotes differentiation with respect to the $z$-component of position vector of the membrane. The force $F$ denotes the (constant) force of the membrane opposing the actin filaments. Assuming a liquid system exterior, the boundary force $F_{b}$ indicates the hydrostatic pressure on the membrane. The random force $\xi_{\text {mem }}$ satisfies

$$
\begin{aligned}
& \left\langle\xi_{\text {mem }}(t) \xi_{\text {mem }}\left(t^{\prime}\right)\right\rangle=2 \gamma_{\text {mem }} k_{\mathrm{B}} T \delta\left(t-t^{\prime}\right), \\
& \left\langle\xi_{\text {mem }}(t)\right\rangle=0 .
\end{aligned}
$$

\subsection{Conditions}

Because we modeled membrane motion in the $z$ direction alone, we impose periodic boundary conditions along $x$ - and $y$-axes as shown in Fig. 2. Simulations were conducted in a unit box with cross-sectional area equal to the membrane $A$. The number of actin filaments $N_{\text {fa }}$ sustaining the membrane was estimated on the basis of the experimental observation [22], and it was reported that the number of actin filaments in $1 \mu \mathrm{m}^{2}$ is approximately $1.4 \times 10^{3}$.
Actin particles were supplied via a particle bath connected to the system at $z=0$. Throughout the simulations, the density of actin particles $\rho$ in the bath was maintained at 0.056 . The boundary force, $F_{b}$, was then estimated as $F_{b}=\rho k_{B} T A$. We arbitrarily set the value of $\gamma_{\text {mem }}$ to be significantly higher than that of actins.

To solve Eqs. (1) and (17), the parameter values were normalized by unit length $\sigma$, unit time $\tau=\sigma^{2} / 2 D$, and unit energy $k_{B} T$. These values are summarized in Table 1. Derivation of non-dimensionalized equations is given in the Appendix.

If the length of the polymerized filament exceeds the critical length at which the filament buckles under the membrane opposing force, the membrane either remains almost stationary or moves backward under the opposing forces. Therefore, the simulation time of calculating the membrane velocity was limited to $100 \tau$, sufficient to polymerize several monomers on each filament.

Because the repulsive interaction between actin particles in the same filament contributes to the effective bending rigidity, the persistence length $L_{p}$ of the filament is not solely determined by $K_{\text {be }}$. Therefore, prior to the numerical experiments, we simulated a non-polymerized filament under thermal fluctuations, and determined $L_{p}=1.3 \times 10^{3} \sigma$ by the tangent correlation method [23]. This value is consistent with the measured persistent length of nonstabilized actin filaments [24]. The stalling force $F_{S}$ at which the mem-

Table 1 Parameters

\begin{tabular}{lll}
\hline Symbol & Value & Description (unit) \\
\hline$\alpha$ & $\{0.17,1.0\} \times 10^{2}$ & Ratio of polymerization time to diffusion time \\
$\rho$ & $5.6 \times 10^{-2}$ & Density of actin particles in particle bath $\left(\sigma^{-3}\right)$ \\
$N_{\mathrm{fa}}$ & 32 & Number of actin filaments \\
$L_{\mathrm{i}}$ & 32 & Initial length of actin filament $(\sigma)$ \\
$A$ & $32^{2}$ & Area of membrane of the unit simulation box $\left(\sigma^{2}\right)$ \\
$F_{b}$ & 60 & Boundary force on the membrane $\left(k_{\mathrm{B}} T / \sigma\right)$ \\
$K_{\mathrm{t}}$ & $2.0 \times 10^{3}$ & Spring constant $\left(k_{\mathrm{B}} T / \sigma\right)$ \\
$K_{\mathrm{be}}$ & $5.0 \times 10^{2}$ & Bending rigidity $\left(k_{\mathrm{B}} T\right)$ \\
$\epsilon$ & $1.878 \times 10^{-1}$ & Energy coefficient in the Lennard-Jones potential $\left(k_{\mathrm{B}} T\right)$ \\
$l_{i i^{\prime}}^{\mathrm{eq}}$ & $5.0 \times 10^{-1}$ & Length of the spring between actins $i$ and $i^{\prime}$ in the stress free state $(\sigma)$ \\
$\theta_{i}^{\text {eq }}$ & $\pi$ & Angle between three actin $i-1, i$, and $i+1$ in the stress free state $(\mathrm{rad})$ \\
$\gamma$ & 2.0 & Friction coefficient of an actin particle $\left(\tau k_{\mathrm{B}} T / \sigma^{2}\right)$ \\
$\gamma_{\mathrm{mem}}$ & $2.0 \times 10^{2}$ & Friction coefficient of membrane $\left(\tau k_{\mathrm{B}} T / \sigma^{2}\right)$ \\
$\phi_{c}$ & $\pi / 3$ & Polymerization angle criterion $(\operatorname{rad})$ \\
$p$ & $\left\{5 \times 10^{5}, 1\right\}$ & Conditional probability of polymerization in case-L and case-H \\
$\Delta t$ & $2 \times 10^{-6}$ & Integration time interval $(\tau)$ \\
$k_{\mathrm{B}} T$ & $4.3 \times 10^{-21}$ & Energy unit $(\mathrm{J}):$ Thermal energy at temperature $T=310 \mathrm{~K}$ \\
$\sigma$ & $5.4 \times 10^{-9}$ & Length unit $(\mathrm{m})$ : actin monomer diameter \\
$\tau$ & $2.9 \times 10^{-6}$ & Time unit $(\mathrm{s}):$ Characteristic time of actin monomer diffusion \\
& & assuming $D=5 \times 10^{-12} \mathrm{~m}^{2} / \mathrm{s}[27]$ \\
\hline & &
\end{tabular}


brane protrusion is halted by filament buckling was estimated as $1.00 \times 10^{2} k_{\mathrm{B}} T / \sigma$ by the Euler column formula [25]. Here, the critical length was approximated as the initial filament length. Equations (1), (11) and (17) were numerically integrated using the Euler method for stochastic differential equations with an integration time $\Delta t$ [26].

\subsection{Limitations of the model}

Because the current model ignores actin filament branching and cross-linking, the simulated actin filaments can be buckled by forces that are smaller than physiologically relevant force. Therefore, we normalized the opposing force $F$ by the stall forces measured in simulations and denoted the normalized force by $\tilde{F}$.

\section{Different polymerization rates result in membranes protruding differently}

To investigate the manner in which the FVR responds to actin polymerization, we simulated a low polymerization rate (case-L; $\alpha=1.0 \times 10^{2}$ ) and a higher polymerization rate (case-H; $\alpha=0.17 \times 10^{2}$ ) and evaluated the FVR in each case.

\subsection{Force-velocity relation (FVR)}

Figure 3 shows that snapshots of the membrane gradually moving upward along the $z$-axis as the growing actin filament pushes it from beneath. The membrane motion fluctuates over small time scales but moves unidirectionally over longer time scales as the membrane fluctuations are occasionally overtaken by the growing actin filament.

The velocity of the membrane protrusion was defined by fitting a linear function to the temporal changes in the membrane position and obtaining the slope. Here, we determined the average velocity of 10 samples. Figure 4 plots the FVRs obtained in both case- $\mathrm{L}$ and $-\mathrm{H}$ together with the theoretical predictions of the BR model $[10,11]$ and quadratic fitting functions. In both cases, fitting to a quadratic function was justified based on Akaike Information Criterion (AIC) [28], in which the lower the AIC, the better the fitting function (see Table 2). The mean velocities $V(F)$ and forces $F$ are normalized by $V(0)$, and the stall force $F_{S}$, respectively. In the case- $\mathrm{L}$ and $-\mathrm{H}, V(0)$ was determined as $1.2 \times 10^{-2}$ and $2.3 \times 10^{-2}(\sigma / \tau)$, respectively, and the respective $F_{S}$ was $1.04 \times 10^{2}$ and $1.01 \times 10^{2}\left(k_{\mathrm{B}} T / \sigma\right)$.

The FVRs significantly differ between the two cases. The FVR is concave up in case- $\mathrm{L}$, as predicted by the BR model, but is concave down in case-H. Because the parameters in both cases were identical except for the polymerization- diffusion ratio $\alpha$, we infer that $\alpha$ drives the qualitative difference between the FVRs.

Under an independent actin filament assumption of the BR model $[10,11]$, the velocity is an exponentially decreasing function of the force opposing the actin filament. This decrease is attributed to reduced polymerization. Therefore, the BR model captures the FVR trend at lower polymerization rates. Conversely, we speculate that the case-H simulations deviate from the predictions of the BR model because the higher polymerization rate encourages multiple filamentmembrane contacts. This idea is explored in the next section.

\subsection{Spatial distribution and forces of actins}

Because membrane protrusion is driven by extension at the plus end of the filament, we investigated the spatial distribution of actin monomers and filaments. This distribution was found to depend on the value of $\alpha$. Figure 5 plots the density distributions of monomers and the plus ends of filaments at $\tilde{F}=0$. In case-L (Fig. 5a), the density of monomers is higher than that of plus ends; moreover, the distribution of plus ends broadly peaks around 1.7 . Because the membrane repels the actins at distances $d_{c} \approx 1.1 \sigma$, most of the filaments make no membrane contact, and fluctuate freely instead, giving rise to the broad peak in the spatial distribution of their plus ends. In case-H (Fig. 5b), the density of plus ends exceeds that of the monomers, and the plus ends cluster at distances around $1.1 \sigma$. Here, the narrow distribution peak is attributed to the contact between the plus ends of the filaments and the membrane.

Because such physical contacts are necessary to sustain the membrane load, we investigated sharing of the membrane load between monomers and filaments. Figure 6 shows the force fraction and the density fraction of monomers. The force fraction expresses the ratio of the force exerted by the filament or monomer as a proportion of the total force. Similarly, the density fraction defines the density ratio of monomers to the total density (obtained by summing the filaments and monomers beneath the membrane within the Lennard-Jones cut-off distance). The mathematical relationship among density distribution, density fraction and force fraction is provided in the Appendix.

The force fraction in case-L indicates that monomers sustain more than half the force on the membrane and that in case- $\mathrm{H}$ is predominantly sustained by filaments. Furthermore, the force fraction of monomers decreases with increasing the force in case- $\mathrm{L}$, but not in case- $\mathrm{H}$. Because the membrane force is counterbalanced by physical contact (i.e., repulsive interactions) between the actins and membrane, these trends reflect the structures built by the actins. Indeed, in case-L, the density and force fractions of monomers follow identical decreasing trends as the membrane force increases. 
(a)

(b)
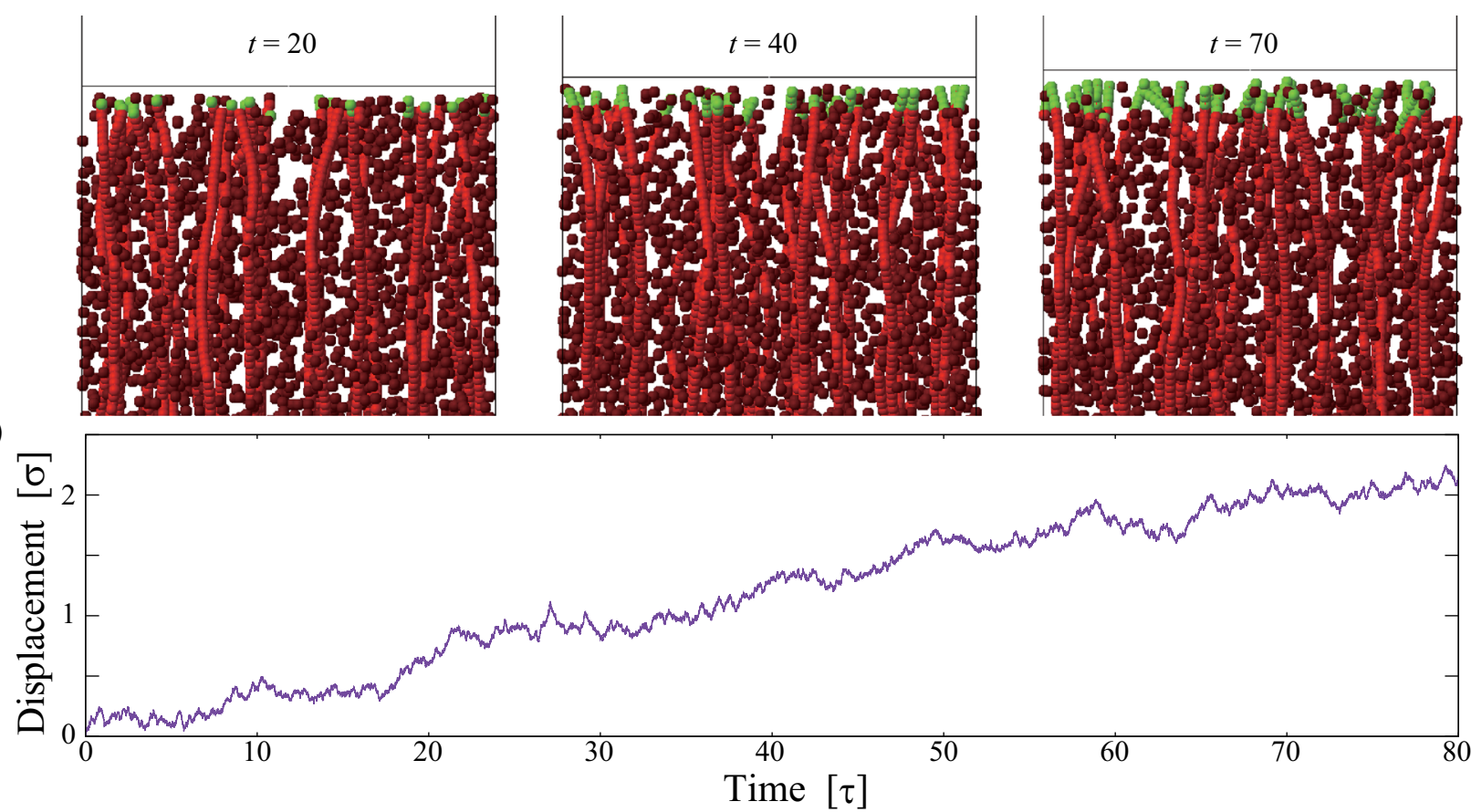

(c)

(d)
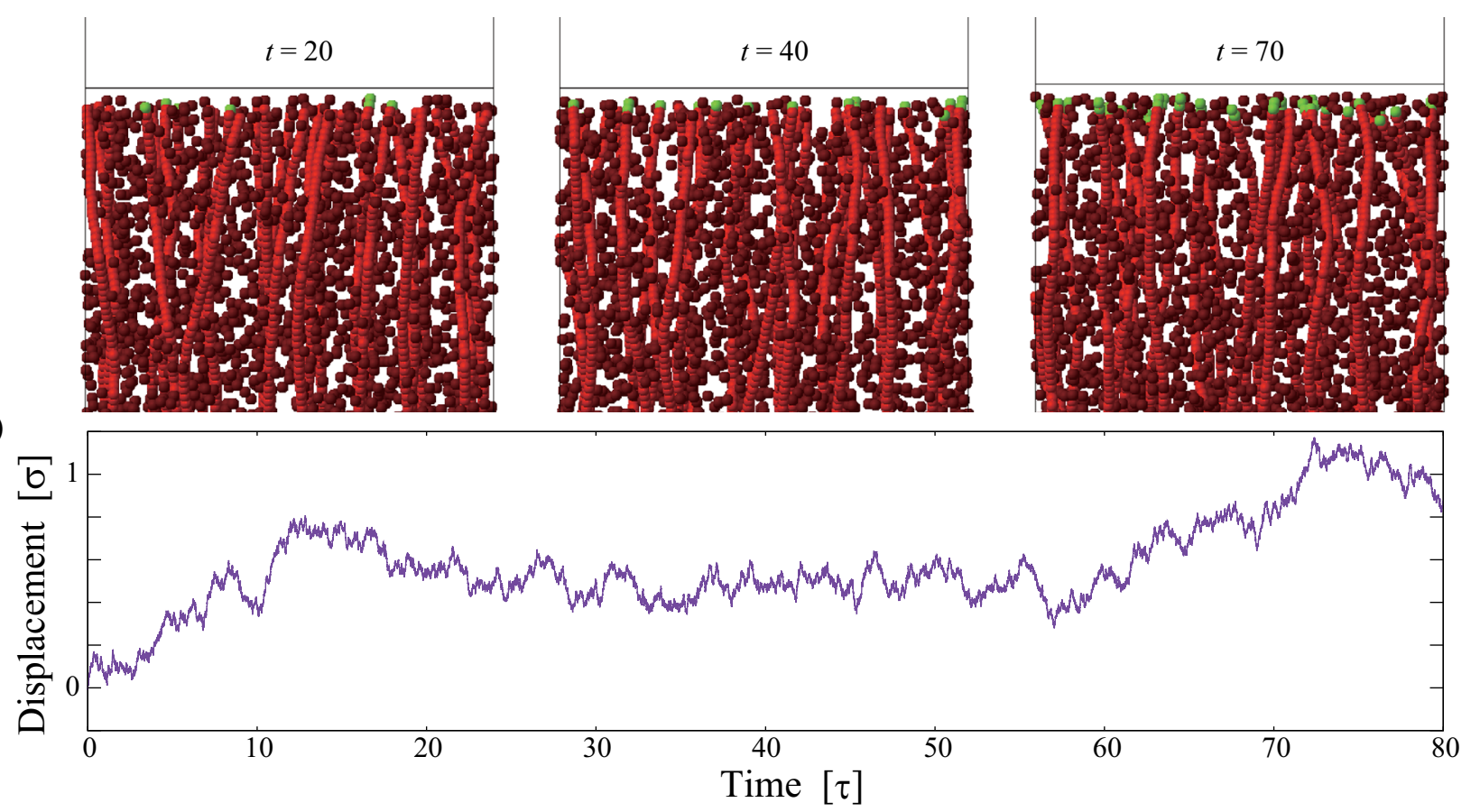

Fig. 3 Snapshots of the simulation results in a case-H and c case-L. The normalized force $\tilde{F}=0.0$. Actin subunits incorporated into actin filaments and actin monomers in cytosol are represented by red and dark red particles, respectively. Green particles represent newly polymerized actin subunits. $i$ along the filament heads indicates the membrane.

\section{Discussion}

By varying $\alpha$, we observed different membrane protrusions and obtained two classes of FVR. Despite ignoring the
The two side lines indicate borders of the simulation box, which are subjected to horizontal periodic boundary conditions. $\mathbf{b}$ and $\mathbf{d}$ show the displacement of the membrane from the initial position as functions of time expressed in unit time $\tau$ in case- $\mathrm{H}$ and case- $\mathrm{L}$, respectively. The displacement is expressed in actin particle size $\sigma$. (Color figure online) branches and cross-linking structures that occur in real polymerizations, the simulations yielded concave-up and -down FVRs. Thus, our simulations suggest an underlying mechanism for the two classes of FVRs. We speculate that the 


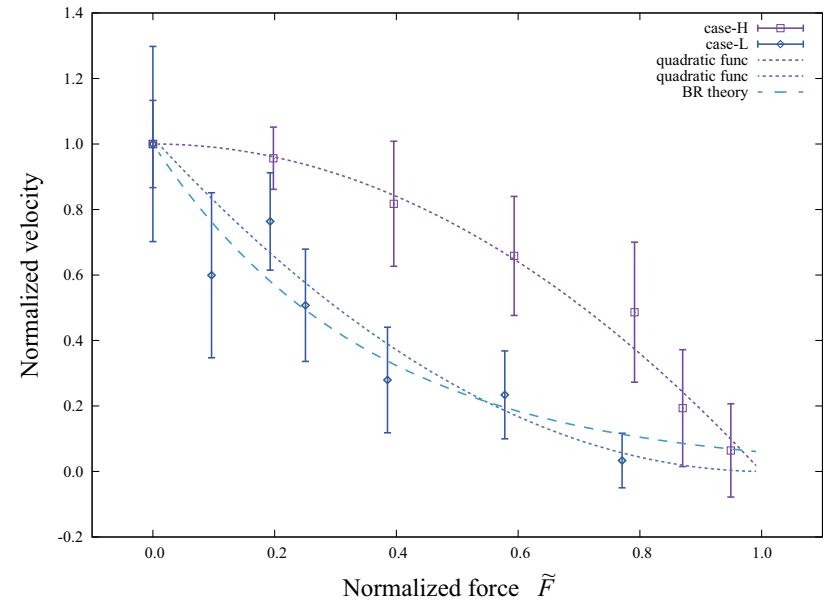

Fig. 4 Simulated force-velocity relation (FVR) in case-L (blue) and case-H (purple), and the theoretical FVR predicted by the Brownian ratchet [11] (dashed cyan curve). Error bars denote the standard deviation. To guide the eye, simulated FVRs are fitted by quadratic functions. In both cases, fitting to a quadratic function has been justified based on AIC. (Color figure online)

Table 2 Akaike Information Criterion (AIC) of the fitting function. Symbol "+" indicates that the term is included in the fitting function

\begin{tabular}{llll}
\hline Case & AIC & Linear term: $\tilde{F}$ & Square term: $\tilde{F}^{2}$ \\
\hline Case-H & -467.3 & + & $\mathrm{n} / \mathrm{a}$ \\
& -486.6 & $\mathrm{n} / \mathrm{a}$ & + \\
& -484.8 & + & + \\
Case-L & -534.1 & + & $\mathrm{n} / \mathrm{a}$ \\
& -514.8 & $\mathrm{n} / \mathrm{a}$ & + \\
& -536.8 & + & + \\
\hline
\end{tabular}

actins are differently distributed in the two cases, which led to different mechanical support of the membrane. These different spatial distributions may contribute to the variant FVRs. Because multiple filaments competed for monomers beneath the membrane, as occurs in vivo and in vitro, we defined the monomer density as the equivalent number of monomers per single filament (i.e. the density of monomers divided by the density of filaments). This is because an effective polymerization is proportional to the number of monomers for a single plus end.

In case-L, the density fraction of monomers decreased with increasing the membrane force, reducing the equivalent monomer density. Thus, the net polymerization also steeply decreased with increasing membrane force and probably caused the concave-up trend of the FVR. The primary reason why increasing membrane force altered the force fraction is because monomers are not bound to each other; thus, filaments join to sustain the membrane gradually with increasing membrane force.

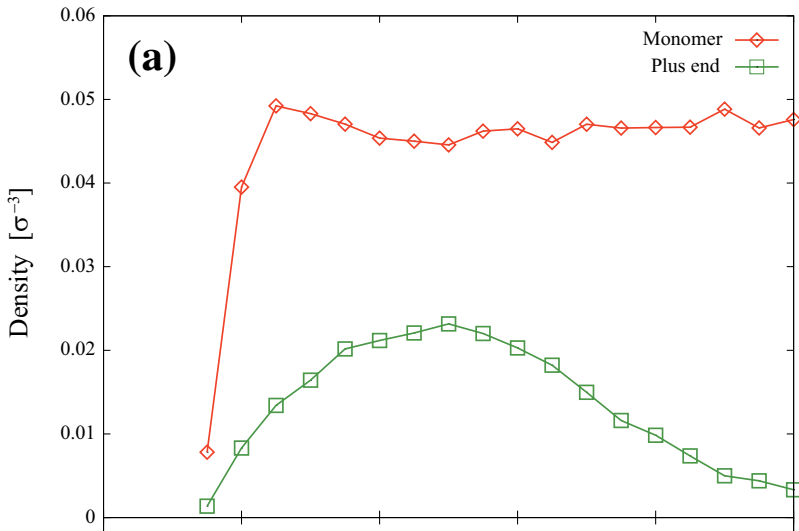

(b)

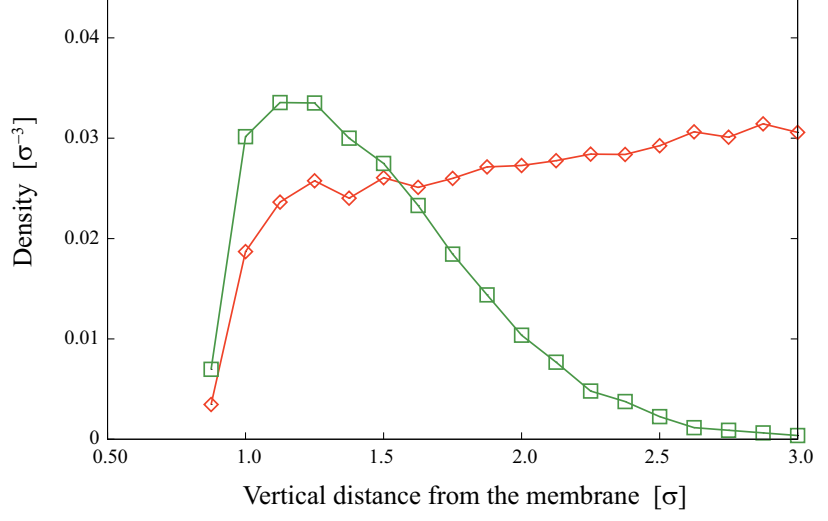

Fig. 5 Vertical distributions of monomers and plus ends of filaments at $\tilde{F}=0$ in (a) case-L, and (b) case-H. The vertical distance indicates the distance along the $z$ axis between the membrane and actin, expressed in the actin particle size $\sigma$

In contrast to case-L, the force fraction in case- $\mathrm{H}$ did not change significantly because the membrane is sustained by the elastic bonds of the filament. Consequently, the monomer density fraction also remains unchanged. Thus, in case- $\mathrm{H}$, the equivalent monomer density did not significantly change with increasing membrane force, leading to a concave-down FVR.

The different spatial distribution of actins can be explained by parameter $\alpha$. Since $\alpha$ was approximately one order of magnitude higher in case- $\mathrm{L}$ than in case- $\mathrm{H}$, the actin monomers were polymerized on the plus ends with much longer duration than in case-H. During this time, monomers could reside and diffuse beneath the membrane. In contrast, the shorter polymerization time in case- $\mathrm{H}$ would deplete the monomers beneath the membrane. Thus, by altering $\alpha$, we can change the spatial distribution of actins and consequently the response of the membrane protrusions to the membrane force.

In a previous BD simulation of the FVR[15], where actin nucleation at the rear side of a disk was triggered by Arp2/3 protein complex, breaking the symmetry of the system driving the unidirectional motion of the disk. The FVR 

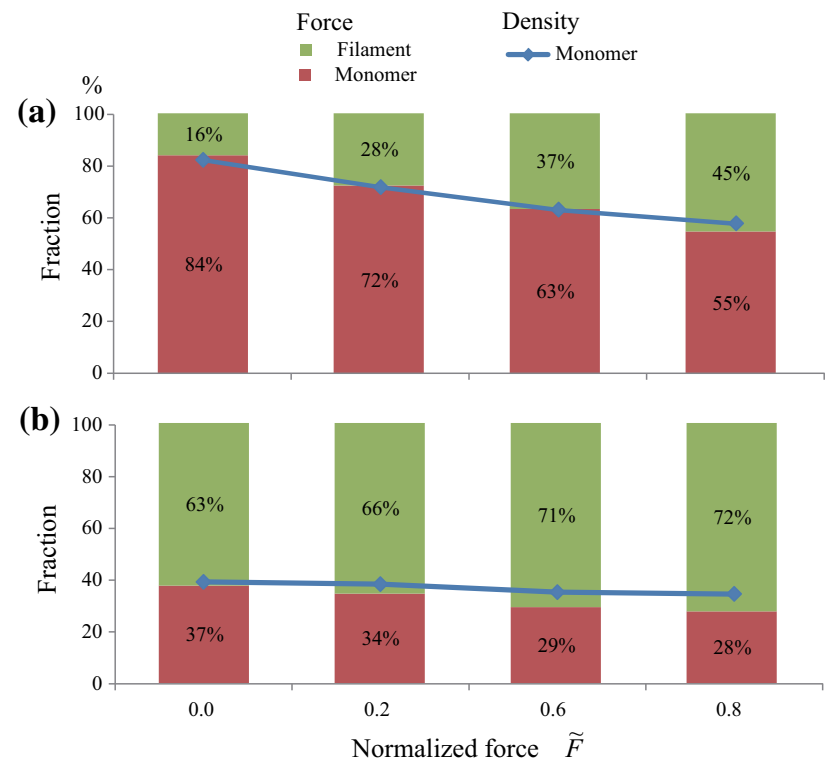

Fig. 6 Force fraction and monomer density fraction vs. membrane force in a case- $\mathrm{L}$, and $\mathbf{b}$ case- $\mathrm{H}$. The filaments and monomers sustain the membrane against the membrane force. The force fraction defines the ratio of the force exerted by the filament or monomer to the total force. The density fraction defines the ratio of the monomer density to the total density (the sum of the filaments and monomers beneath the membrane within the Lennard-Jones cut-off distance)

obtained in [15] was concave down, and the actin density in the filament sharply peaked near the disk. The density of free monomers was not shown. The results of [15] qualitatively agree with those of our case-H simulations, suggesting that the spatial distribution of actin filaments largely governs the FVR trends. However, the two studies exhibit slight quantitative differences in the FVR under small loads. The velocity of the disk is almost independent of load in [15], but depends on the load in our study. We speculate that direct nucleation of actin polymerization at the rear side of the disk or branching filaments suppresses the velocity decrease of the disk under small loads. Thus, to better understand the FVR, BD simulations should account for the fine structure of the molecular system involved in membrane protrusion. Then, the manner in which these actin-related and protein-binding molecules influence the FVR should be investigated.

Acknowledgments This study was partially supported by KAKENHI (25702025) and the Platform for Dynamic Approaches to Living System from the Ministry of Education, Culture, Sports, Science and Technology, Japan.

\section{Appendix 1: Non-dimensionalized equations}

In this section we provide the derivation of the nondimensionalized versions of Eqs. (1) and (17). Normalizing parameter values by the unit length $\sigma$, the unit time $\tau=$ $\sigma^{2} / 2 D$, and the unit energy $k_{B} T$, Eq. (1) is rewritten as

$\gamma \frac{\sigma}{\tau} \hat{\mathbf{v}}_{i}=-\frac{1}{\sigma} \hat{\nabla}_{i} k_{\mathrm{B}} T \hat{U}_{i}-\frac{1}{\sigma} \hat{\nabla}_{i} k_{\mathrm{B}} T \hat{U}_{i}^{\mathrm{m}}+\frac{k_{\mathrm{B}} T}{\sigma} \hat{\boldsymbol{\xi}}$.

Here, $\hat{\imath}$ indicates a non-dimensionalized parameter, operator or function. Dividing both sides of Eq. (20) by $k_{\mathrm{B}} T / \sigma$, the non-dimensionalized version of Eq. (1) is derived as

$\hat{\gamma} \hat{\mathbf{v}}_{i}=-\hat{\nabla}_{i} \hat{U}_{i}-\hat{\nabla}_{i} \hat{U}_{i}^{\mathrm{m}}+\hat{\boldsymbol{\xi}}$

where,

$\hat{\gamma}=\frac{\gamma \sigma^{2}}{\tau k_{\mathrm{B}} T}$.

Substituting $\tau=\sigma^{2} / 2 D$ and $D=k_{\mathrm{B}} T / \gamma$ into the right hand side of Eq. (22), $\hat{\gamma}=2$ is obtained.

The non-dimensionalized version of Eq. (17) is derived by the same procedure and is expressed as

$\hat{\gamma}_{\text {mem }} \hat{v}_{\text {mem }}=-\sum_{k} \hat{\nabla} \hat{U}_{k}^{\mathrm{m}}-\hat{F}-\hat{F}_{b}+\hat{\xi}_{\text {mem }}$,

where

$$
\begin{aligned}
\hat{\gamma}_{\mathrm{mem}} & =\frac{\gamma_{\mathrm{mem}} \sigma^{2}}{\tau k_{\mathrm{B}} T}, \\
& =\frac{2 \gamma_{\mathrm{mem}}}{\gamma} .
\end{aligned}
$$

In Eqs. (24) and (25), we used $\tau=\sigma^{2} / 2 D$ and $D=k_{\mathrm{B}} T / \gamma$.

\section{Appendix 2: Relationship among density distribu- tion, density fraction and force fraction}

The total density distribution $\rho_{\mathrm{t}}\left(r_{z}\right)$ is defined by

$\rho_{\mathrm{t}}\left(r_{z}\right)=\rho_{\mathrm{m}}\left(r_{z}\right)+\rho_{\mathrm{f}}\left(r_{z}\right)$.

Here, $\rho_{\mathrm{m}}\left(r_{z}\right)$ and $\rho_{\mathrm{f}}\left(r_{z}\right)$ denote the density distribution of free actin particles and the density distribution of actin particles constituting filaments at a vertical distance $r_{z}$, respectively. The vertical distance indicates the distance along the $z$-axis between the membrane and actin. The density distribution of filaments can be decomposed into a series composed of the density distribution of the plus end, the density distribution of particle adjacent to the plus end, next particle, and so on. The density distribution of the plus end is illustrated in Fig. 5. The density fraction of monomers, $\lambda_{\text {dens }}$, is defined by

$\lambda_{\text {dens }}=\frac{\int_{0}^{d_{c}} \rho_{\mathrm{m}}\left(r_{z}\right) \mathrm{d} r_{z}}{\int_{0}^{d_{c}} \rho_{\mathrm{t}}\left(r_{z}\right) \mathrm{d} r_{z}}$. 
The total force $F$ exerted on the membrane is expressed by

$F=-A \int_{0}^{d_{c}} \mathrm{~d} r_{z} \rho_{\mathrm{t}}\left(r_{z}\right) \frac{\partial U_{L J}\left(r_{z}\right)}{\partial r_{z}}$

Substituting Eq. (26) into the right hand side of Eq. (28), $F$ is rewritten as

$F=F_{\mathrm{m}}+F_{\mathrm{f}}$,

where,

$F_{\mathrm{m}}:=-A \int_{0}^{d_{c}} \mathrm{~d} r_{z} \rho_{\mathrm{m}}\left(r_{z}\right) \frac{\partial U_{L J}\left(r_{z}\right)}{\partial r_{z}}$,
$F_{\mathrm{f}}:=-A \int_{0}^{d_{c}} \mathrm{~d} r_{z} \rho_{\mathrm{f}}\left(r_{z}\right) \frac{\partial U_{L J}\left(r_{z}\right)}{\partial r_{z}}$.

$F_{\mathrm{m}}$ and $F_{\mathrm{f}}$ indicate the forces exerted by the free actin and actin constituting filaments, respectively. The force fraction of the monomer and the force fraction of the filament are defined by $F_{\mathrm{m}} / F$ and $F_{\mathrm{f}} / F$, respectively.

\section{References}

1. Small JV, Herzog M, Anderson K (1995) Actin filament organization in the fish keratocyte lamellipodium. J Cell Biol 129:12751286

2. Small JV, Stradal T, Vignal E, Rottner K (2002) The lamellipodium: where motility begins. Trends Cell Biol 12:112-120

3. Lauffenburger DA, Horwitz AF (1996) Cell migration: a physically integrated molecular process. Cell 84:359-369

4. Goldberg MB (2001) Actin-based motility of intracellular microbial pathogens. Microbiol Mol Biol Rev 65:595-626

5. Marcy Y, Prost J, Carlier MF, Sykes C (2004) Forces generated during actin-based propulsion: a direct measurement by micromanipulation. Proc Natl Acad Sci USA 101:5992-5997

6. Borisy GG, Svitkina TM (2000) Actin machinery: pushing the envelope. Curr Opin Cell Biol 12:104-112

7. Keren K, Pincus Z, Allen GM, Barnhart EL, Marriott G, Mogilner A, Theriot JA (2008) Mechanism of shape determination in motile cells. Nature 453:475-480

8. Lacayo CI, Pincus Z, VanDuijn MM, Wilson CA, Fletcher DA, Gertler FB, Mogilner A, Theriot JA (2007) Emergence of largescale cell morphology and movement from local actin filament growth dynamics. PLoS Biol 5:2035-2052

9. Prass M, Jacobson K, Mogilner A, Radmacher M (2006) Direct measurement of the lamellipodial protrusive force in a migrating cell. J Cell Biol 174:767-772

10. Peskin CS, Odell GM, Oster GF (1993) Cellular motions and thermal fluctuations: the Brownian ratchet. Biophys J 65:316-324
11. Mogilner A, Oster G (1996) Cell motility driven by actin polymerization. Biophys J 71:3030-3045

12. Mogilner A, Oster G (2003) Force generation by actin polymerization II: the elastic ratchet and tethered filaments. Biophys $\mathbf{J}$ 84:1591-1605

13. Atilgan E, Wirtz D, Sun SX (2006) Mechanics and dynamics of actin-driven thin membrane protrusions. Biophys J 90:65-76

14. Schreiber CH, Stewart M, Duke T (2010) Simulation of cell motility that reproduces the force-velocity relationship. Proc Natl Acad Sci USA 107:9141-9146

15. Lee KC, Liu AJ (2009) Force-velocity relation for actinpolymerization-driven motility from Brownian dynamics simulations. Biophys J 2:1295-1304

16. Blanchoin L, Boujemaa-Paterski R, Sykes C, Plastino J (2014) Actin dynamics, architecture, and mechanics in cell motility. Physiol Rev 94(1):235-263

17. Goley ED, Rammohan A, Znameroski EA, Firat-Karalar EN, Sept D, Welch MD (2010) An actin-filament-binding interface on the Arp2/3 complex is critical for nucleation and branch stability. Proc Natl Acad Sci USA 107(18):8159-8164

18. Smith BA, Daugherty-Clarke K, Goode BL, Gelles J (2013) Pathway of actin filament branch formation by Arp2/3 complex revealed by single-molecule imaging. Proc Natl Acad Sci USA 110(4):1285-1290

19. Risca VI, Wang EB, Chaudhuri O, Chia JJ, Geissler PL, Fletcher DA (2012) Actin filament curvature biases branching direction. Proc Natl Acad Sci USA 109:2913-2918

20. Shimada Y, Adachi T, Inoue Y, Hojo M (2009) Coarse-grained modeling and simulation of actin filament behavior based on Brownian dynamics method. Mol Cell Biomech 6:161-173

21. Inoue Y, Deji T, Shimada Y, Hojo M, Adachi T (2010) Simulations of dynamics of actin filaments by remodeling them in shear flows. Comput Biol Med 40:876-882

22. Abraham VC, Krishnamurthi V, Taylor DL, Lanni F (1999) The actin-based nanomachine at the leading edge of migrating cells. Biophys J 77(3):1721-32

23. Li X, Lehman W, Fischer S (2010) The relationship between curvature, flexibility and persistence length in the tropomyosin coiled-coil. J Struct Biol 170:313-318

24. Isambert H, Venier P, Maggs AC, Fattoum A, Kassab R, Pantaloni D, Carlier MF (1995) Flexibility of actin filaments derived from thermal fluctuations. Effect of bound nucleotide, phalloidin, and muscle regulatory proteins. J Biol Chem 270:11437-11444

25. Mogilner A, Rubinstein B (2005) The physics of filopodial protrusion. Biophys J 89:782-795

26. Mannella R, Palleschi V (1989) Fast and precise algorithm for computer simulation of stochastic differential equations. Phys Rev A 40:3381-3386

27. McGrath JL, Tardy Y, Dewey CF Jr, Meister JJ, Hartwig JH (1998) Simultaneous measurements of actin filament turnover, filament fraction, and monomer diffusion in endothelial cells. Biophys $\mathbf{J}$ 75:2070-2078

28. Akaike H (1973) Information Theory and an Extension of the Maximum Likelihood Principle. In: Petrov BV, Csaki F (eds) 2nd International Symposium on Information Theory. Akad Kiado, Budapest, pp 267-281 